\title{
Finding the Best Path in a Binary Block Interference Network
}

\author{
Edwin Soedarmadji*, Robert J. McEliece \\ California Institute of Technology \\ MC 136-93, Pasadena, CA, USA \\ edwin@systems.caltech.edu,rjm@systems.caltech.edu
}

\begin{abstract}
A binary Block Interference Channel (BIC) is model of binary channels with memory that allows for a mathematically tractable computation of channel capacity. One can easily imagine interconnecting such channels into a network that allows point-to-point communication between any two nodes in the network. Given a pair of network nodes, finding the path with the highest capacity is quite trivial if we can assume that all participating nodes in any path connecting the two nodes can perform coding at arbitrary complexity such that at each link capacity is achieved. However, even if the complexity assumption is not taken into account, in most real-life networks (such as the current Internet), only a minimum amount of coding is performed at the link layer. In most networks, coding is performed five or six layers up in the OSI network model, i.e., on either the presentation or the application layer. Under such realistic circumstances, finding the path with the highest capacity is no longer trivial. In this paper, we propose a solution based on a modified version of the Dijkstra's Algorithm.
\end{abstract}

\section{INTRODUCTION}

Many modern communication networks can be mathematically described as networks of binary communication channels. In many scenarios, errors occuring along these channels can be approximately modeled as random outages.

However, often these channels exhibit the so-called memory effect. For a period following an error, a channel often experiences a higher than normal rate of error. For example, when a packet received at an intermediate router completely fills up the router's queue, any subsequent packet (and hence the binary symbols therein) will be dropped until the congestion is cleared up. For this reason, we often say that network errors are "bursty." Clearly, in many applications, accurate behavior and performance analysis has to take into account the fact that channel errors exhibits memory.

The Gilbert Channel Model (GCM) [2] is one of the simplest models for analyzing the engineering performance of channels with memory. Despite (or we may say because of) its simplicity, the GCM has been widely used in analyzing a wide variety of real-life networks [3].

For example, GCM is used to analyze the performance of slotted ALOHA over fading communications channels [4], correlated loss over TCP/IP networks [5], real-time wireless communications [6], and many others.

For many different types of wireless fading channels, the GCM is a very attractive alternative to sophisticated models such as Hidden Markov models (HMMs) [7] because compared to the HMM's, the GCMs are analytically more tractable. This desirable property leads to a polynomial-time algorithm for finding the path with the best error performance (QoS) in a network of Gilbert channels [8].
However, the GCM does not lend itself for a tractable information-theoretic analysis of channel capacity. The mathematical expression for Shannon capacity of a single Gilbert channel is a set of recursive equations in the various underlying channel parameter [9]. The complexity only gets worse when the capacity analysis is extended to network paths, which is crucial for finding the path with the best Shannon capacity.

For this very reason, McEliece and Stark introduced the novel concept of Block Interference Channel (BIC) [10]. A Gilbert channel models a channel with memory as two (or a finite number of) multiplexed channels that are selected at each time $t$ based on the current state $S_{t}$ of a Markov chain with two (or the same number of) states.

A BIC model simplifies this by requiring that the above Markov chain have balanced transition probabilities, which immediately means the state sequence is now an i.i.d sequence. In addition, in a BIC, the Markov chain is not updated everytime $t$ increases, but rather at a lower clock cycle (which in this paper we denote by $k$ ) every $m$ cycles.

These simplifications allow one to compute a closed-form expression for Shannon capacity upper-bound in cases where the side information $S_{k}$ is available to the receiving end. It is shown in [10] that in the limit of large $m$, this side information can be readily and reliably inferred by the receiver. In such cases, the upper bound becomes tight. In this paper, we focus our analysis on a special class of BICs that contain two underlying Binary Symmetric Channels (BSCs) as described in Example 1a of [10]: one lossless BSC denoted by $\Delta_{0}$, and the other BSC $\Delta_{\delta}$ with crossover probability $\delta$.

At this point, we also would like to point out that if we can assume that all participating nodes in any path connecting the two nodes can perform coding at arbitrary complexity such that at each link capacity is achieved, then given any pair of network nodes, the task of finding the path with the highest capacity is quite trivial. One could first compute the capacity for each link, assign these capacities on the corresponding link, and run a (min,max) [11] algorithm on the network.

However, even if the unrealistic arbitrary-complexity assumption is not taken into account, in most real-life networks (such as the current implementation of the Internet), only a minimum amount of coding is performed at the physical link layer. In most networks, coding is not performed until the symbols reach five or six layers up in the OSI network model, i.e., on either the presentation or the application layer.

Under such realistic circumstances, finding the path with the highest capacity is no longer trivial. Each possible path constitutes a separate channel with its own Shannon capacity, and therefore the (min,max) algorithm cannot be used. 
In this paper, we propose a solution based on a modified version of the Dijkstra's Algorithm (DA) [12]. The solution involves defining a rule to combine parameters of adjacent BICs into parameters of a composite BIC, and a rule to compare two different BICs. Using these rules, the algorithm computes path parameters and compares them to find the path with the most desirable BIC parameters. The algorithm presented in this paper can be used in networks where GCM (and hence BIC) is used to analyze the underlying links, and wherever shortest path routing is used.

Finally, before we begin our main discussion in the next section, we provide our view of why an information-theoretic routing optimization in a network of BICs is a very relevant topic. With the advent of Universal Mobile Telecommunications Systems (UMTS) networks where multimedia (especially speech) and data packets will have to coexist in the underlying common Wideband Code-Division Multiple Access (WCDMA) networks, the GCM has become more important.

The recent UMTS recommendations for QoS measures is based on a set of user satisfaction assessments of individual speech and data sessions [13].

For speech services, user satisfaction drops significantly in the presence of long spans (in packets) of service outage. For data services, disruption and termination comes from successive retransmissions.

These new QoS measures require the next level of approximation of behavioral analysis that is not available from system outage probability analysis (which inherently assumes zero correlation between the outages) commonly found in CDMA literature [14], [15]. Recently, detailed analysis (in the context of QoS and capacity) of WCMDA [16], [17] incorporates correlated outage behavior by using GCM. In the next level of analysis that incorporates coding and capacity, the simplified BIC models would prove indispensable.

This paper is organized as follows. In Section II, we provide a concise mathematical formulation of the problem and define the notations. In Section III we explain the so-called Generalized Dijkstra's Algorithm that plays the central role in our results. Finally, in Section IV we discuss our conclusion and some suggestions for future research.

\section{FORMULATION AND NOTATION}

We model our network as a digraph $G=(V, E)$, and denote by $V, E$, and $\Pi$ the node, edge and path sets of $G$, respectively. Denote by $s$ and $d \in V$ the network source and destination nodes, and by $\Pi \subset \Pi$ the set of all network paths from $s$ to $d$. A path $\pi \in \Pi$ whose nodes $V_{\pi} \subset V$ are connected by $E_{\pi} \subset E$ is denoted by either $\left\langle v_{0}, \ldots, v_{J}\right\rangle$ or $\left\langle e_{1}, \ldots, e_{J}\right\rangle$. The symbol $\left\langle v_{i}, v_{i+1}\right\rangle$ denotes the edge (path) connecting the two (non-) adjacent nodes $v_{i}$ and $v_{i+1}$.

Each edge $e_{i} \in E$ has its own edge weight which is a 2-D vector $\boldsymbol{\lambda}_{i} \in \Lambda$. The elements $\left(\epsilon_{i}, \delta_{i}\right)$ of $\boldsymbol{\lambda}_{i}$ are the parameters $\epsilon_{i}$ and $\delta_{i}$ of the BIC for a given $e_{i}$. The special value $\lambda=\infty$ denotes the absence of connection between two nodes.

For all $e_{i} \in E$ we assume that $\boldsymbol{\lambda}_{i}$ are fixed, and that $0 \leq$ $\epsilon_{i} \leq 1$ and $0 \leq \delta_{i} \leq \frac{1}{2}$. We also assume that each BIC $e_{i}$ consists of two BSC's: (1) a noiseless BSC denoted by $\Delta_{0}$, and (2) a BSC with crossover probability $s_{i}$ denoted by $\Delta_{\delta_{i}}$.
Consider a binary sequence $x=\left\{x_{0}, x_{1}, x_{2}, \ldots\right\}$ transmitted from $s$ to $d$. The BIC assumes that $x$ can be grouped together into $X=\left\{X_{0}, X_{1}, X_{2}, \ldots\right\}$ where $X_{k}=$ $\left\{x_{k m+0}, x_{k m+1}, \ldots, x_{k m+m-1}\right\}$. We assume that each channel imposes a zero transmission delay. Therefore, we can think of $k$ as the time variable for the entire network.

For each edge $e_{i} \in E$, define a sequence of i.i.d binary random variables $S_{i}=\left\{S_{i 0}, S_{i 1}, S_{i 2}, \ldots\right\}$. The random variables are such that the probability of $S_{i k}=0$ (indicating that $e_{i}$ is representing $\Delta_{0}$ ) is $\epsilon_{i}$. Conversely, the probability of $S_{i k}=1$ (indicating that $e_{i}$ is representing $\Delta_{\delta_{i}}$ ) is $1-\epsilon_{i}$.

Denote by $C: \Lambda \rightarrow \mathcal{C} \equiv[0,1]$ the function that measures the capacity of an edge $e \in E$ with a channel parametrized by $\lambda \in \Lambda$. For convenience, denote by $\beta: \Pi \rightarrow \Lambda$ the function that measures the parameter $\boldsymbol{\lambda}_{\pi}$ (or $\boldsymbol{\lambda}_{i}$ ) of a given path $\pi$ (or a given edge $e_{i}$ ) and the shorthand notation $C(e)=C(\beta(e))$.

The addition operator can be defined in $\Lambda$. Let us denote this operation by $\oplus$. If $\boldsymbol{\lambda}_{1}=\beta\left(e_{1}\right), \boldsymbol{\lambda}_{2}=\beta\left(e_{2}\right)$, and $\pi=\left\langle e_{1}, e_{2}\right\rangle$, then we say that $\boldsymbol{\lambda}_{\pi}=\boldsymbol{\lambda}_{1} \oplus \boldsymbol{\lambda}_{2}$. These expressions imply that $\oplus$ and $\Lambda$ have to obey some algebraic properties (such as closure), which we will discuss in the next section.

Having defined $\oplus$, we can now express the path channel parameter $\boldsymbol{\lambda}_{\pi}$ in terms of $\boldsymbol{\lambda}_{i}$ using a generalized summation

$$
\lambda_{\pi}=\bigoplus \lambda_{i}
$$

Let us call the pair $(\Lambda, \oplus)$ the $\mathbf{B}$ algebra equipped with the associated $\beta$ and $C$ functions. Note that $\oplus, \beta$ and $C$ are not enough to calculate the path $\pi^{*}$ with the highest capacity. For this, we need to able to compare edge weights and path lengths (using their corresponding capacities). Mathematically, we need a total order operator $\preceq$ acting on $\Lambda$ and $\mathcal{C}$ to evaluate expressions like $c_{\pi} \preceq c_{\pi^{\prime}}$ and $\boldsymbol{\lambda}_{\pi} \preceq \boldsymbol{\lambda}_{\pi^{\prime}}$. With a definition of $\preceq$, we can write the following expression:

$$
\begin{aligned}
\boldsymbol{\lambda}^{*} & =\min _{\pi}\left\{\boldsymbol{\lambda}_{\pi} \mid \pi \in \Pi\right\} \\
c^{*} & =\min _{\pi}\left\{c_{\pi} \mid \pi \in \Pi\right\} \\
\pi^{*} & =\operatorname{argmin}_{\pi}(C(\pi))
\end{aligned}
$$

Next, we define $\oplus$ operating on our BICs. First, note that the blocks $X_{i}$ are independent, and thus we can think of each $X_{i}$ block as a symbol drawn from a $q$-ary alphabet where $q=2^{m}$. Further, as shown in [10], each BIC can be thought of as a $2^{m}$-ary symmetric channel with channel error probability $\epsilon\left(1-(1-\delta)^{-m}\right)$. Let $q$-SC $(p)$ be the abbreviation for a $q$-ary symmetric channel with channel error probability $p$.

It is easy to prove that a series combination of a $q-\mathrm{SC}\left(p_{1}\right)$ and a $q-\mathrm{SC}\left(p_{2}\right)$ is equivalent to a $q-\mathrm{SC}(p)$. The equations for $p$ and the Shannon capacity of $q-\mathrm{SC}(p)$ are:

$$
\begin{aligned}
p_{1} & =\epsilon_{1}\left(1-\left(1-\delta_{1}\right)^{-m}\right) \\
p_{2} & =\epsilon_{2}\left(1-\left(1-\delta_{2}\right)^{-m}\right) \\
p & =p_{1} \oplus p_{2} \\
p_{1} \oplus p_{2} & =1-\left(1-p_{1}\right)\left(1-p_{2}\right)-\frac{1}{q-1}\left(p_{1} p_{2}\right)
\end{aligned}
$$

Each BIC on $e_{i}$ is the same, whether it is parametrized by a 2-D vector $\boldsymbol{\lambda}_{i}$ or by a scalar $p_{i}$, which is obviously preferable. In this new parametrization, $\boldsymbol{\lambda}_{i} \equiv p_{i}=\epsilon_{i}\left(1-\left(1-\delta_{i}\right)^{-m}\right)$ and thus the space $\Lambda$ now is just $[0,1]$. 
Denote by $C_{q}(p)$ (or $C(p)$ if $q$ is fixed) the Shannon capacity of a $q-\operatorname{SC}(p)$. Note that the argument $p$ can now correspond to the parameter of a single channel corresponding to an edge $e$, or from a path constructed from several such edges. From [18], $C(p)$ is given by:

$$
C(p)=1+(1-p) \log _{q}(1-p)+p \log _{q}(p)-p \log _{q}(q-1)
$$

We are now ready to define the $\preceq$ operator which we will use to compare different $\lambda$ 's. The definition of $\preceq$ in $\mathcal{C} \equiv[0,1]$ is quite obvious: it is just the standard $\leq$ operator. It is not clear if we can order the members of $\Lambda$ in a similar manner.

First, let us make a statement that is true regardless of the exact representation of $\boldsymbol{\lambda}$. If we let $\boldsymbol{\lambda}_{1}=\beta\left(e_{1}\right)$ and $\boldsymbol{\lambda}_{2}=$ $\beta\left(e_{2}\right)$, then in $\mathbf{B}$, the operator $\preceq$ is defined as follows:

$$
\boldsymbol{\lambda}_{1} \preceq \boldsymbol{\lambda}_{2} \quad \text { iff } \quad C\left(\boldsymbol{\lambda}_{1}\right) \preceq C\left(\boldsymbol{\lambda}_{2}\right) .
$$

The above definition simply orders the channels based on their Shannon capacity. Consequently, given the source node $s$ and the destination node $d$, we define the best path $\pi^{*}$ as the path with the highest Shannon capacity.

However, since $\lambda$ 's are just $p$ 's, and for a fixed $q$, the capacity $C_{q}(p)$ is a monotonically increasing function of $p$ with a peak at $p=\frac{q-1}{q}$. Therefore, the definition of $\preceq$ for $\Lambda$ boils down to the standard $\leq$ operator on $[0,1]$. The coupling of the $\mathbf{B}$ algebra with the $\preceq$ operator induces a metric which we shall call the $\mathbf{B}$ metric.

In the next sections, we will show that elements of the parameter space $\Lambda$ and the corresponding $\mathbf{B}$ algebra are suitable as inputs to a modified version of the Dijkstra's Algorithm, which is known to have a time-complexity of $O\left(V^{2}\right)$ [19].

\section{Generalized DiJKstra’s Algorithm}

The problem of finding the optimal path with minimum length (measured in the metric of choice - in our paper the B metric) in a network represented by a graph $G$ can be solved using the Generalized Dijkstra's Algorithm (GDA) below [12]:

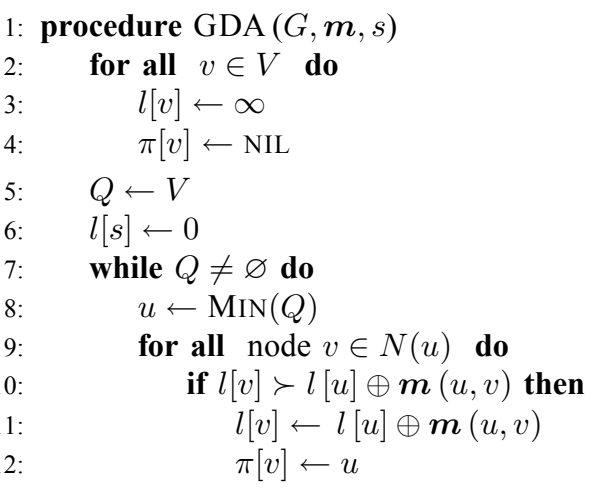

The GDA is practically identical to the Dijkstra's Algorithm (DA) except for the relaxation step, where $\oplus$ and $\preceq$ operators act on a general metric space $\mathcal{M}$ (instead of the equivalent step in DA, where + and $\leq$ operators act on $\mathbb{R}$ ).

On line 9, $N(u)$ denotes the set of all nodes adjacent to $u$. The argument $\boldsymbol{m}$ is the edge lengths in $G$ each of which is an element in $\mathcal{M}$, and $\boldsymbol{m}(u, v)$ is the length of $\langle u, v\rangle$. Lines 10-12 perform the relaxation step of the GDA.
This step depends on the definitions of $\mathcal{M}, \oplus$, and $\preceq$. If the GDA (in)correctly returns the path in $G$ with minimum length measured in $\mathcal{M}$, then $(\mathcal{M}, \oplus)$ and $\preceq$ are said to be (in) compatible with the GDA. The following is the required properties for compatibility:

Proposition 1 An algebra $\mathbf{A}=(\mathcal{M}, \oplus)$ and a total order $\preceq$ is compatible with the GDA if and only if it satisfies all the properties in the set denoted by $\mathbf{P}$ below:

$\mathbf{P 1}$ is a commutative monoid, that is, for $a, b, c \in \mathcal{M}$ :

- $\mathcal{M}$ is closed under $\oplus: a \oplus b \in \mathcal{M}$;

- $\oplus$ is associative : $a \oplus(b \oplus c)=(a \oplus b) \oplus c$;

- 0 is the identity : $a \oplus 0=0 \oplus a=a$;

- $\oplus$ is commutative : $a \oplus b=b \oplus a$.

P2 There exists $\infty \in \mathcal{M} \mid a \oplus \infty=\infty \oplus a=\infty$.

P3 $\preceq$ is a total order on $\mathcal{M}$, i.e., $\preceq$ is :

- reflexive: $a \preceq a$;

- anti-symmetric: if $a \preceq b$ and $b \preceq a$ then $a=b$;

- transitive: if $a \preceq b$ and $b \preceq c$ then $a \preceq c$;

- total: for every $a, b \in \mathcal{M}$ either $a \preceq b$ or $b \preceq a$.

P4 There exists the least element 0 that satisfies $0 \preceq a$.

P5 $a \oplus c \prec b \oplus c$ if $a \prec b$ and $c \in \mathcal{M}-\{\infty\}$.

PROOF: Refer to [12] for a complete proof.

THEOREM 2 The algebra $\mathbf{B}=(\Lambda, \oplus)$ and $\preceq$ satisfy all the properties in $\mathbf{P}$, and thus compatible with the GDA.

Proof: In the following derivation, let $a, b$, and $c$ be the channel parameters of three BICs associated with the edges $e_{1}, e_{2}, e_{3} \in E$, and $\infty$ denotes the lack of connection.

$$
a \oplus b=1-(1-a)(1-b)-(a b) /(q-1)
$$

P1 Except for closure, the monoid properties can be proven with algebraic manipulation of equation (4). Recall that $\mathcal{P}=$ $[0,1] \cup \infty$. Closure is obvious if $a, b \in[0,1]$. If $a=\infty$ or $b=\infty$, then by the definition of $\infty$ as a special designation for the absence of connection between two nodes, then we must have $a \oplus b=\infty$.

P2 The proof is derived from closure on $\infty$.

P3 The proof follows the definition of $\mathcal{P}$.

P4 Same as above.

P5 The proof is obvious if $b=\infty$. However, if $b \neq \infty$, then by substituting $a, b$, and $c$ into (4) we obtain the two values $a \oplus c$ and $b \oplus c \in[0,1]$ given by:

$$
\begin{aligned}
& a \oplus c=1-(1-a)(1-c)-a c /(q-1) \\
& b \oplus c=1-(1-b)(1-c)-b c /(q-1) .
\end{aligned}
$$

Since both are in $[0,1]$, the order $\prec$ is just $<$, and the expression $a \oplus c \prec b \oplus c$ is equivalent to the inequality $(a \oplus c)-(b \oplus c)<0$, which can be simplified into:

$$
(1-c q /(q-1))(a-b)<0
$$

If $c \leq \frac{q-1}{q}$, then $0<(1-c q /(q-1)) \leq 1$, and $(a-b)<0$ (because $a \prec b$ and $b \neq \infty$ ). Thus, the above inequality is true, and we have proven all the properties in $\mathbf{P}$.

CORROLARY 3 The function $C(p)$ is a non-decreasing function of $p$, which means that the path with minimum $p$ (from the GDA) is also the path with minimum $C(p)$.

PROOF: The proof follows from our definition for $\preceq$ : 


\section{CONCLUSION AND Discussion}

The preceding results provide us with a practical method to find the best path that maximize Shannon capacity in a network of binary Block Interference channels. The method is based on the Generalized Dijkstra's Algorithm (GDA), which requires the edge metric to obey a certain set of necessary and sufficient conditions. The Shannon capacity of each path is computed and compared against other paths.

Our approach characterizes each edge (and the corresponding BIC) by its error parameters, which are themselves probabilistic density parameters. Using these density parameters as edge lengths, we compute any path lengths by an appropriate combination of edge lengths according to the laws of probability. This approach fully preserves the stochastic nature of the problem (instead of simply reducing the stochastic edge weights to proxy deterministic values such as their averages). Future work includes simulating or experimentally verifying the theoretical results presented herein, and considering the other examples presented in [10].

\section{ACKNOWLEDGMENTS}

This work was supported by the Caltech Lee Center for Advanced Networking and NSF Grant No. CCF-0514881.

\section{REFERENCES}

[1] Sobrinho, J.L., "Algebra and algorithms for QoS path computation and hop-by-hop routing in the Internet," IEEE/ACM ToN., vol. 10, pp. 541550, August 2002.

[2] Gilbert, E. N., "Capacity of a burst-noise channel," Bell Syst. Tech. J., vol. 39, pp. 1253-1265, Sep. 1960 .

[3] Swarts, J.S., Ferreira, H.C., "On the evaluation and application of Markov channel models in wireless communications," Vehicular Technology Conference, 1999. VTC 1999 - Fall. IEEE VTS 50th
[4] Tan, H.H., Hung, S.V., "Performance analysis of slotted ALOHA over fading communications channels," INFOCOM '90. Ninth Annual Joint Conference of the IEEE Computer and Communication Societies. Proceedings., IEEE

[5] Zorzi M., Rao R.R., "The Effect of Correlated Errors on the Performance of TCP” IEEE Communications Letters, Vol. 1, No.. 5, September 1997

[6] Lee K.K., Chanson S.T., "Packet loss probability for real-time wireless communications," IEEE Trans. Veh. Technol., vol. 51, no. 6, pp. 15691575, Nov. 2002.

[7] Turin, W. and Van Nobelen, R., "Hidden Markov modeling of fading channels," Proc. IEEE Veh. Technol. Conf., May 1998, pp. 1234-1238.

[8] Soedarmadji E., "Reliability measure of networks with time-correlated edge failures," Submitted to the 28th International Conference on Distributed Computing Systems (ICDCS 2008), Beijing 2008.

[9] Mushkin, M., Bar-David, I., "Capacity and coding for the Gilbert-Elliot channels," IEEE Transactions on Information Theory, Vol.IT-35, No.6, pp.1277-1290; Nov 1989

[10] McEliece R.J., Stark W.E., "Channels with Block Interference," IEEE Trans. on Inf. Theory, Vol.IT-30, No.1, pp.44-53, January 1984.

[11] Aji S.M., McEliece R.J., "The generalized distributive law," IEEE Transactions on Information Theory, Vol.46, No.2 pp.325-343; March 2000 .

[12] Sobrinho, J.L., "Algebra and algorithms for QoS path computation and hop-by-hop routing in the Internet," IEEE/ACM Transaction on Networking., vol. 10, pp. 541-550, August 2002.

[13] ETSI, "Selection Procedures for the choice of radio transmission technologies of the UMTS," Universal Mobile Telecommunications System (UMTS); TR 101112 V3.2.0 (1998-04), Annex B.

[14] Gilhousen K.S., Jacobs I.M., Padovani R., Viterbi A.J., Weaver L.A.Jr., and Weatley C.E., "On the capacity of a cellular CDMA system," IEEE Trans. Veh. Technol., vol. 40, no. 2, pp. 303-312, May 1991.

[15] Evans J.S., Everitt D., "On the teletraffic capacity of CDMA cellular networks," IEEE Trans. Veh. Technol., vol. 48, no. 1, pp. 153-166, Jan. 1999.

[16] Elshabrawy, T., Le-Ngoc, T., "Capacity of future WCDMA networks supporting multimedia services," Selected Areas in Communications, IEEE Journal on, Volume 24, Issue 1, Jan. 2006 Page(s):54 - 64

[17] Elshabrawy, T., Le-Ngoc, T., "Gilbert channel approximation for downlink performance evaluation of WCDMA systems," Vehicular Technology Conference, 2005. VTC 2005 Fall. 2005 IEEE 62nd, Volume 1, 28-25 Sept., 2005 Page(s):382 - 386

[18] Shokrollahi A., "Capacity-Approaching codes on the q-ary symmetric channel for large q," Proceedings of IEEE ISIT '04, pp.204-208; San Antonio, TX, USA; Oct. 2004

[19] Cormen, T.H.; Leiserson, C.E.; Rivest, R.L., Introduction to Algorithms, MIT Press, MA and McGraw-Hill, NY, 1990. 